\title{
OPEN Integrated transcriptomic and metabolomic analysis provides insight into the regulation of leaf senescence in rice
}

\author{
Jiao Xue ${ }^{1,2}$, Dongbai Lu ${ }^{1,2}$, Shiguang Wang ${ }^{1}$, Zhanhua Lư ${ }^{1}$, Wei Liu ${ }^{1}$, Xiaofei Wang ${ }^{1}$, \\ Zhiqiang Fang ${ }^{1}$ \& Xiuying $\mathrm{He}^{1 \bowtie}$
}

Leaf senescence is one of the most precisely modulated developmental process and affects various agronomic traits of rice. Anti-senescence rice varieties are important for breeding application. However, little is known about the mechanisms underlying the metabolic regulatory process of leaf senescence in rice. In this study, we performed transcriptomic and metabolomic analyses of the flag leaves in Yuenong Simiao (YN) and YB, two indica rice cultivars that differ in terms of their leaf senescence. We found 8524 genes/204 metabolites were differentially expressed/accumulated in YN at 30 days after flowering (DAF) compared to 0 DAF, and 8799 genes/205 metabolites were differentially expressed in YB at 30 DAF compared to 0 DAF. Integrative analyses showed that a set of genes and metabolites involved in flavonoid pathway were significantly enriched. We identified that relative accumulation of PHENYLALANINE AMMONIA-LYASE (PAL), CINNAMATE 4-HYDROXYLASE (C4H), 4-COUMAROYL-COA LIGASE (4CL), CHALCONE SYNTHASE (CHS) and CHALCONE ISOMERASE (CHI) in $Y N 30 / 0$ was higher than that in $Y B 30 / 0$. Three flavonoid derivatives, including phloretin, luteolin and eriodictyol, showed lower abundances in YB than in YN at 30 DAF. We further revealed a MYB transcription factor, which is encoded by OsR498G0101613100 gene, could suppress the expression of $\mathrm{CHI}$ and $\mathrm{CHS}$. Our results suggested a comprehensive analysis of leaf senescence in a view of transcriptome and metabolome and would contribute to exploring the molecular mechanism of leaf senescence in rice.

Leaf senescence, an orderly process of degradation and transformation, is the last stage of leaf development and is controlled by complex internal factors such as hormones, genetics, and external gene network regulatory factors, including light, water, temperature, mineral elements, and microorganisms $\mathrm{s}^{1-3}$. Leaf senescence plays important roles in regulating nutrient distribution and improving the ability of plants to adapt to the environment. Through the process of senescence, leaves transfer nutrients to seeds or fruits at the end of development, providing necessary energy for offspring, seed germination and early growth of seedlings ${ }^{4}$. During senescence, leaf cells undergo dramatic changes in cell metabolism, structure, and gene expression. The most striking feature of these changes is that the decomposition of chlorophyll during chloroplast denaturation results in the yellowing of leaves, followed by hydrolysis of macromolecules such as lipids, proteins and nucleic acids, which in turn leads to separation of mitochondria and nuclei and ultimately cell death ${ }^{5}$. Together, the identification and characterization of hundreds of senescence-associated genes (SAGs) in plants and senescence-related mutants constitute one of the best approaches for understanding leaf senescence at the molecular level ${ }^{5-7}$. The leaf senescence database (LSD) also provides comprehensive information concerning SAGs and their corresponding mutants ${ }^{8}$.

Transcriptome sequencing is an important method for obtaining gene expression data in organisms ${ }^{9}$. Highthroughput profiling of transcripts is also an efficient method for investigating the process of plant leaf senescence, as has been demonstrated for Arabidopsis thaliana, rice, tobacco, soybean, wheat, cotton, and maize $\mathrm{e}^{10-15}$. However, metabolites are the basis and direct manifestation of an organism's phenotype. Thus, metabolomics provides a global physiochemical view of cellular status during leaf senescence. By detecting the major carbohydrate and nitrogen metabolite markers of tobacco leaves at different developmental stages, researchers identified

${ }^{1}$ Guangdong Key Laboratory of New Technology in Rice Breeding, Rice Research Institute, Guangdong Academy of Agricultural Sciences, Guangzhou 510640, People's Republic of China. ${ }^{2}$ These authors contributed equally: Jiao Xue and Dongbai Lu. ${ }^{\varpi}$ email: hexiuying@gdaas.cn 
a sink-to-source transition at a particular stage in which an accumulation of carbohydrates and a depletion of both organic and inorganic nitrogen stores were observed ${ }^{16}$. By determining changes in chemical composition during leaf senescence in Arabidopsis thaliana, researchers also found that the C, Cr, Cu, Fe, K, Mo, N, P, S and $\mathrm{Zn}$ mobilized from senescing leaves ${ }^{17}$. In recent years, studies combining transcriptomics and metabolomics have been successfully used to research the biosynthesis pathway of medicinally important active ingredients in plants ${ }^{18,19}$, to study the fruit ripening and disease resistance of tomato ${ }^{20,21}$, to elucidate molecular mechanism underlying rice drought tolerance ${ }^{22}$, and so on. New genes related to senescence could still be discovered, and the metabolic pathways in which they are involved concerning the regulation of senescence should be explored by multi-omic approaches.

Rice is one of the most important crop species in the world and is the staple food for more than half of the global population ${ }^{23}$. Rice yield and other agronomic traits are of concern to scientists. In the process of breeding, whether the process of functional leaves turning from green to yellow is stable and smooth in the late-mature stage of rice is also a concern of breeders. This stage is accompanied by the degradation of chlorophyll, pigment deposition and rapid changes in metabolite content. Leaves, especially flag leaves, which are the main functional organs of photosynthesis in rice, are the primary contributor to the accumulation of dry matter in grains. Therefore, premature leaf senescence is one of the main factors affecting rice yield stability. The leaf senescence database currently contains more than 180 SAGs experimentally identified in rice ${ }^{8}$; these SAGs are involved in chloroplast degradation, hormones and transcription factors associated with photosynthesis, the energy metabolism pathway, nitrogen mobilization and so on. However, the biochemical basis and molecular regulatory mechanisms underlying these processes in rice are not clear.

Yuenong Simiao (YN) is a major rice cultivar in South China and exhibits a strong anti-senescence phenotype at the late-mature stage. To explore the underlying molecular mechanisms of the anti-senescence of YN, we performed transcriptomic and metabonomic analyses of the flag leaves of YN as well as YB, another rice variety that exhibits an early-senescence phenotype but is similar to YN in terms of flowering time, resistance, and yield. We identified 8524 differentially expressed genes (DEGs) in YN and 8799 DEGs in YB. By integrating transcriptome and metabolomic data, we found that a set of genes and metabolites involved in flavonoid pathway may exhibit different expression patterns among YN and YB. Interestingly, we found that one MYB TF, OsR498G0101613100, could inhibit the expression of CHALCONE ISOMERASE (CHI) and CHALCONE SYNTHASE (CHS), which were indispensable for the flavonoid pathway in rice. The findings can help to understand the regulatory mechanisms of flavonoid biosynthesis and may be useful for the further research on anti-senescence in rice.

\section{Results}

High-quality Yuenong Simiao rice exhibits a significant anti-senescence phenotype. Yuenong Simiao is a major rice variety with good quality in South China, with a total planting area of more than 200,000 ha. This variety has high yields, strong disease resistance and other excellent traits. In addition, the leaf colour changes slowly from green to yellow in the late stage of development, which is an important characteristic selected by breeders. To study the characteristics of leaf colour transformation, we chose YB as a control variety. The flowering period of $\mathrm{YB}$ was the same as that of $\mathrm{YN}$, but the leaf colour changed from green to yellow and withered rapidly. Given that the leaf colour change from green to yellow is a notable indicator of senescence of plants, we performed detailed physiological, biochemical and molecular assays to determine whether YN has anti-senescence traits.

After flowering, YN plants exhibited a delayed green-to-yellow phenotype, becoming senescent approximately 10 days later than the YB plants did (Fig. 1a, b). In addition, compared with the YB plants, the YN plants underwent slower chlorophyll degradation. The most pronounced difference between the YN and YB plants was that, at 30 days after flowering (DAF), the chlorophyll content of YB decreased dramatically, while that of YN showed a slower decrease (Fig. 1c). Darkness is one of the most powerful external stimuli of leaf senescence and is used frequently as an effective method of simulating synchronous senescence ${ }^{24}$. Leaf segments of YN and YB were subjected to darkness for 5 days. We found that, compared with YB, YN showed significantly delayed dark-induced leaf senescence (Fig. 1d). Reactive oxygen species (ROS) constitute one of the factors that promote senescence ${ }^{25}$. We used 3,3'-diaminobenzidine (DAB) staining to examine $\mathrm{H}_{2} \mathrm{O}_{2}$ levels in the two rice cultivars, and the results showed that the YN leaves displayed less $\mathrm{H}_{2} \mathrm{O}_{2}$ accumulation than the YB leaves did (Fig. 1e). Similarly, chlorophyll degradation-related genes (Red Chlorophyll Catabolite Reductase 1 (RCCR1), NYC1 Like $(N O L)$ and Non Yellow Colouring $3(N Y C 3)$ ) and three other senescence-associated genes (SAGs) (Oryza sativa NAC-like, activated by apetala 3/pistillata (OsNAP), Osh36 and OsI57) were expressed at higher levels in the fully expanded leaves of YB than in those of YN (according to the qPCR results) (Fig. 1f). These results clearly demonstrated that $\mathrm{YN}$ is an elite rice cultivar with a strong anti-senescence phenotype at the filling stage.

Transcriptional analysis reveals a set of differentially expressed genes involved in leaf senescence. To understand the transcriptional landscape in leaves during senescence, we performed an RNA-seq analysis of the flag leaves of YN and YB. We evaluated the flag leaves at 0 DAF and 30 DAF. Pearson's correlation coefficients between biological replicates indicated high reproducibility of the biological replicates and the array platform (Supplemental Table S1). Nearly $80 \%$ of the transcripts were covered more than $60 \%$ of the reads, indicating substantial coverage of the transcriptome (Supplemental Table S1). A snapshot of the transcriptome was generated by clustering all the samples using principal component analysis (PCA). Consistent with their distinct developmental programmes and cultivars, the samples of different cultivars at different developmental stages were clustered (Supplemental Figure S1). Compared to those in the 0 DAF samples, the transcripts in 30 DAF samples had 8524 DEGs in YN and 8799 DEGs in YB, with their expression levels differing by twofold or more. Compared to YB, the transcripts in YN had 6311 DEGs at 0 DAF and 3230 DEGs at 30 DAF (Supplemen- 
a

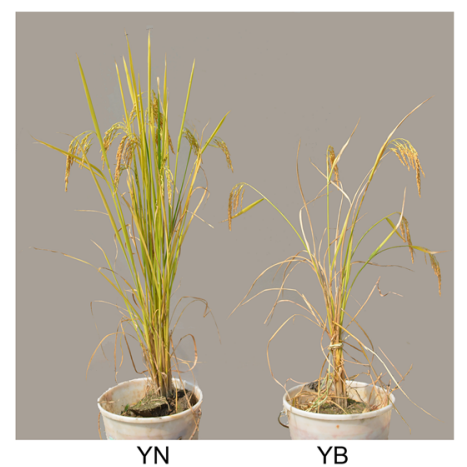

b

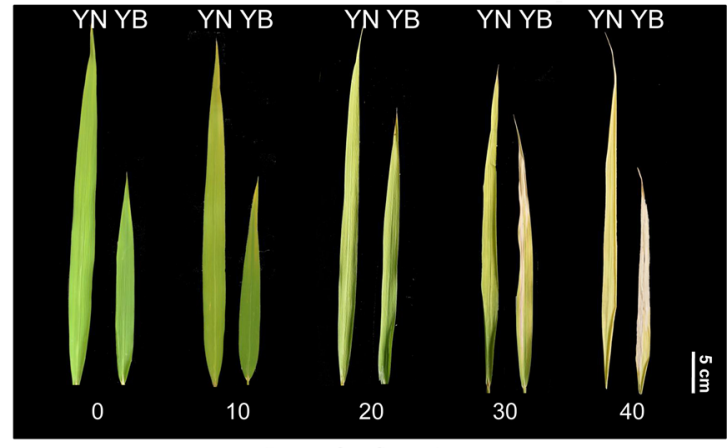

Days after flowering
C
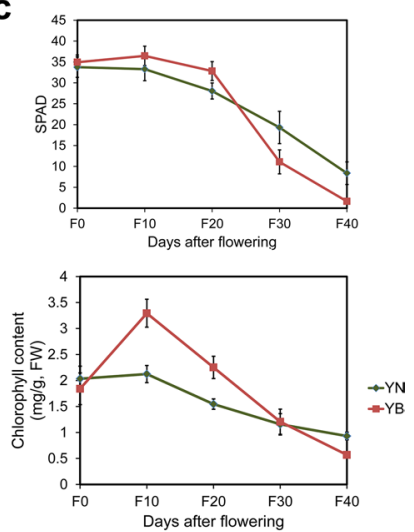

d

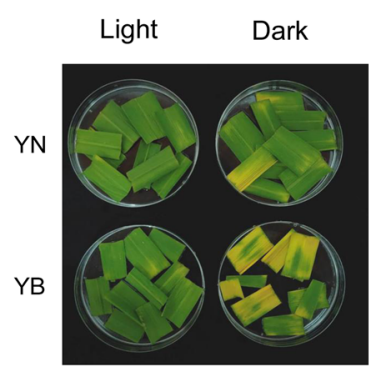

e YN YB

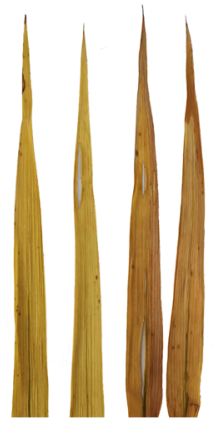

f
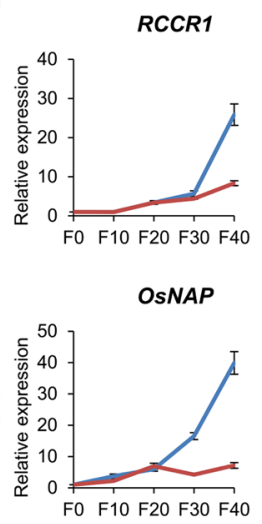

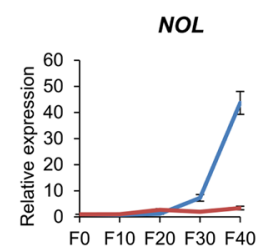

Osh36

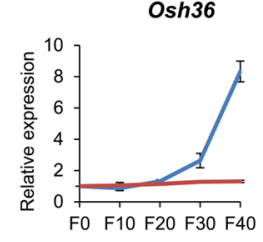

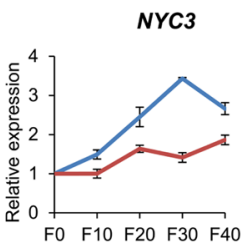

OsI57 $\quad-\mathrm{YN}$

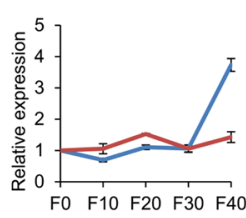

Figure 1. Phenotypes of two rice cultivars. (a) Phenotypes of YN and YB at $30 \mathrm{DAF}$. YN is shown on the left, and YB in shown on the right. (b) Phenotypes of flag leaves of YN and YB plants at different stages after flowering. Bars $=5 \mathrm{~cm}$. (c) SPAD values and chlorophyll contents of flag leaves of $\mathrm{YN}$ and YB plants after flowering. The values are the means \pm SDs of 3 measurements. $F W$ fresh weight. (d) Detached flag leaves from YN and YB plants at the flowering stage were incubated in water for 5 days in darkness. (e) DAB staining of flag leaves of YN and YB at 30 DAF. (f) Relative expression of RCCR1, NOL, NYC3, OsNAP, Osh36 and OsI57 in YN and YB flag leaves at different stages after flowering. The values are the means \pm SDs of 3 repeats.

tal Table S2). GO and KEGG analyses were performed for the DEGs, revealing information on the annotations of genes involved in biological processes, molecular functions and cellular components (GO analysis) and those involved in cellular processing, environmental information processing, genetic information processing, metabolism and organismal systems (KEGG analysis) (Fig. 2a, b, Supplemental Figure S2, Supplemental Table S3). We analysed the top 15 metabolic pathways that were significantly enriched and found that genes differentially expressed in YN at $30 \mathrm{DAF}$ compared to $0 \mathrm{DAF}$ were significantly enriched in the following: photosynthesisantenna proteins, porphyrin and chlorophyll metabolism, arachidonic acid metabolism, glutathione metabolism, flavonoid biosynthesis, the mRNA surveillance pathway, benzoxazinoid biosynthesis, carbon metabolism, the pentose phosphate pathway, ribosome biogenesis in eukaryotes, isoflavonoid biosynthesis, aminoacyl-tRNA biosynthesis, fructose and mannose metabolism, fatty acid elongation, taurine and hypotaurine metabolism. Similarly, and found that genes differentially expressed in YB at 30 DAF compared to 0 DAF were significantly enriched in the following: the mRNA surveillance pathway, glutathione metabolism, photosynthesis-antenna proteins, isoflavonoid biosynthesis, fructose and mannose metabolism, arachidonic acid metabolism, the pentose phosphate pathway, flavonoid biosynthesis, carbon metabolism, porphyrin and chlorophyll metabolism, glycerophospholipid metabolism, MAPK signalling pathway-plant, alpha-linolenic acid metabolism, carotenoid biosynthesis, and glycerolipid metabolism (Fig. 2c, d, Supplemental Table S3). Interestingly, YN exhibited more upregulated genes at $30 \mathrm{DAF}$ than at $0 \mathrm{DAF}$, and YB exhibited more downregulated genes. We identified 3016 genes whose expression was upregulated and 452 genes whose expression was downregulated only in YN0 versus YN30, and we identified 1217 genes whose expression was upregulated and 2526 genes whose expression was downregulated only in the YB0 versus YB30 comparison. A common set of 4851 (3103 upregulated and 1748 downregulated) genes was detected between the YN0 versus YN30 and YB0 versus YB30 comparisons (Fig. 2e). Taken together, these results suggest that at $30 \mathrm{DAF}$, when $\mathrm{YN}$ is still in a period of strong gene expression and metabolism, the gene expression in YB is attenuated (Fig. 2a, b), which is consistent with YB showing an increased-senescence phenotype.

Transcription factors play important regulatory roles in plant growth and development. In total, 385 and 448 transcription factors (TFs) were differentially expressed in YN and YB, respectively (Fig. 2f, g, Supplemental 
a

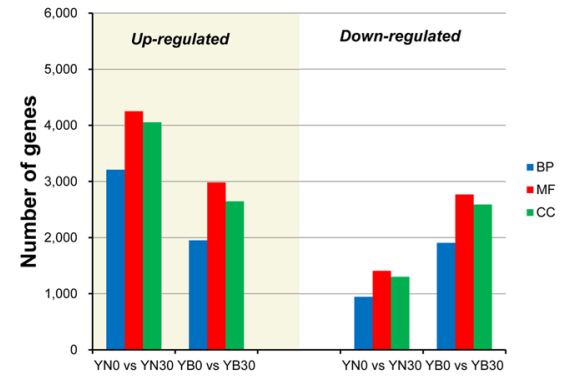

C

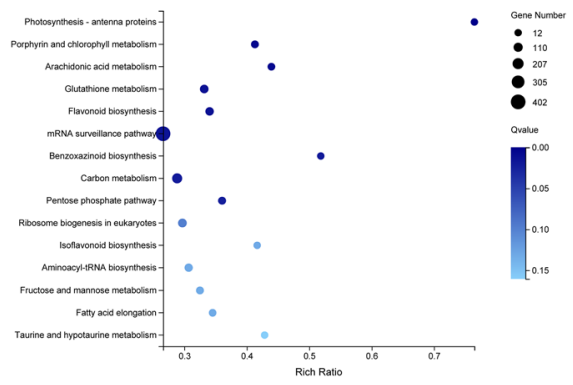

b

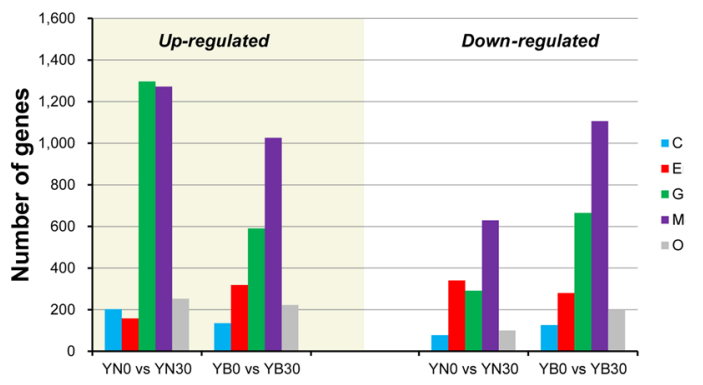

d

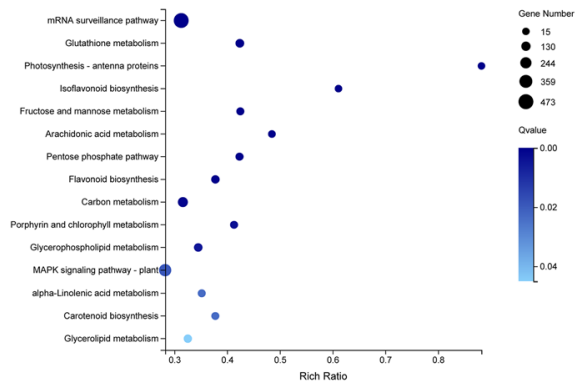

g

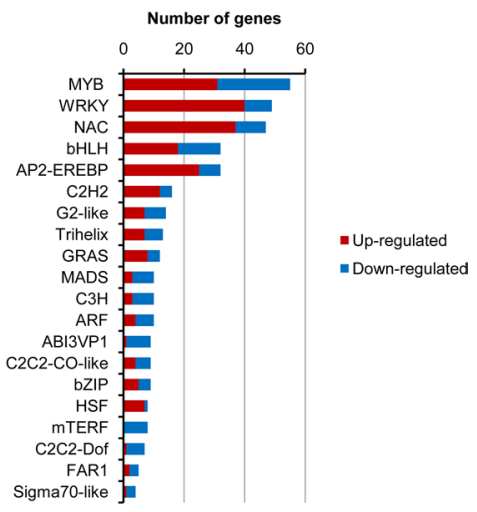

Figure 2. Analysis of differentially expressed genes in YN and YB. (a) GO functions of DEGs. BP biological process, $M F$ molecular function, $C C$ cellular component. (b) KEGG analysis of DEGs. $C$ cellular processes, $E$ environmental information processing, $G$ genetic information processing, $M$ metabolism, $O$ organismal systems. (c) KEGG enrichment analysis of DEGs in the YN0 versus YN30 comparison. The modules show only the top 15 pathways with the most significant enrichment. (d) KEGG enrichment analysis of the DEGs in the YB0 versus YB30 comparison. The modules show only the top 15 pathways with the most significant enrichment. (e) Venn diagrams of the number of DEGs in 30 DAF plants compared with 0 DAF plants. The red characters represent numbers of genes whose expression is upregulated, and the blue characters represent numbers of genes whose expression is downregulated. (f) Analysis of differentially expressed putative transcription factors associated with the DEGs in the YN0 versus YN30 comparison. (g) Analysis of differentially expressed putative transcription factors associated with the DEGs in the YB0 versus YB30 comparison.

Table S4), 112 TFs of which were downregulated and 273 TFs of which were upregulated in YN plants at 30 DAF compared to 0 DAF, and 185 TFs and 263 TFs were downregulated and upregulated, respectively, in YB plants at 30 DAF compared 0 DAF.

Metabolome profiling analysis reveals a set of changes in metabolites involved leaf senescence. To investigate the main metabolic changes reflecting the variation during leaf senescence and to evaluate their putative role in metabolic signalling, a widely targeted metabolome method was used to quantify primary and secondary metabolites identified at different leaf stages. Flag leaves of YN and YB sampled at 0 DAF and 30 DAF were subjected to UPLC-ESI-MS/MS analysis. In this work, 512 and 510 metabolites were identified and quantified in YB and YN, respectively (Supplemental Table S5). PCA showed that YB and YN were clearly separated in the PC1 $\times$ PC2 score plots (Supplemental Figure S3). Of the metabolites, 204 markedly changed in YN at 30 DAF compared to 0 DAF, in which the accumulation of 121 metabolites was upregulated and that of 
a

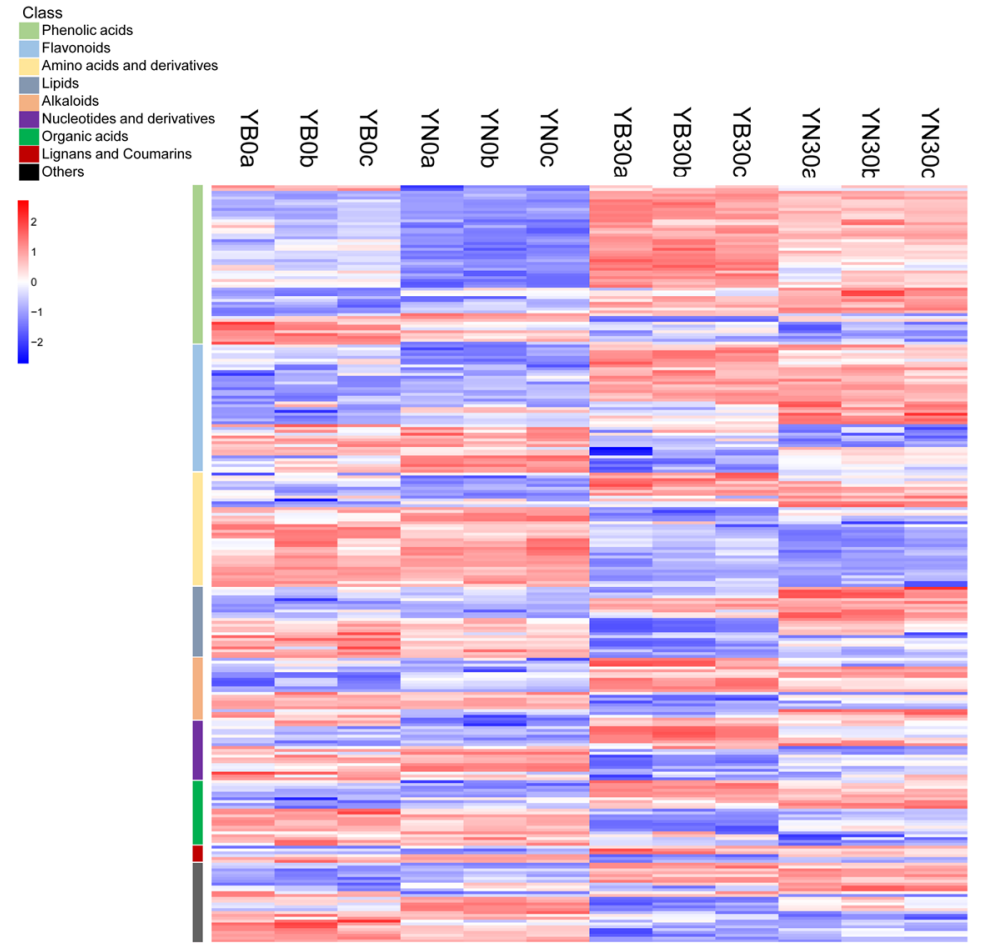

b

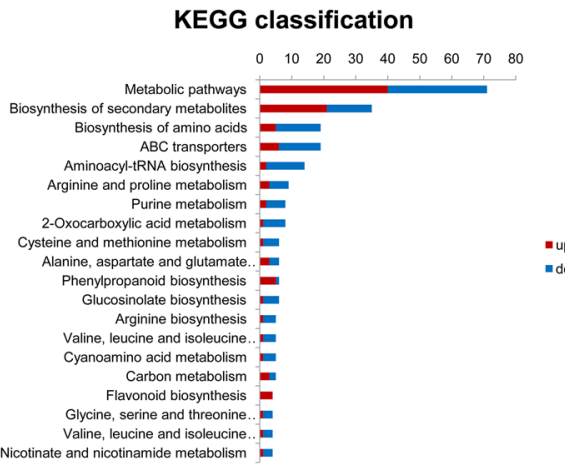

C

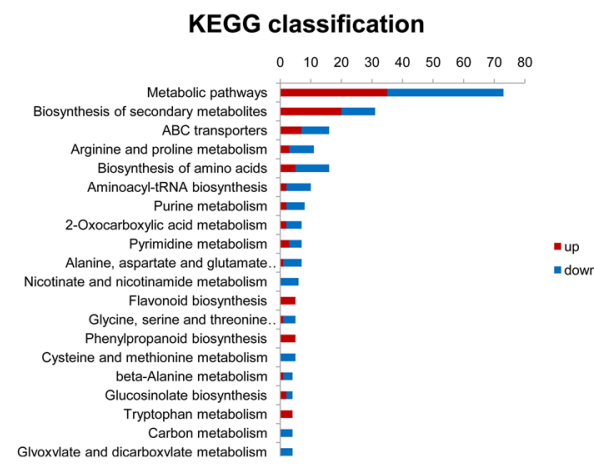

Figure 3. Analysis of differentially accumulated metabolites. (a) Heat map analysis of differentially accumulated metabolites. The data are homogenized by row. (b) KEGG classification of differentially accumulated metabolites in the YN0 versus YN30 comparison. The abscissa represents the number of metabolites. (c) KEGG classification of differentially accumulated metabolites in the YB0 versus YB30 comparison. The abscissa represents the number of metabolites.

83 metabolites was downregulated. A total of 205 metabolites markedly changed in YB at 30 DAF compared to 0 DAF, with the accumulation of 111 and 94 metabolites was upregulated and downregulated (Supplemental Table S5). Cluster analysis was carried out for different metabolic components, including phenolic acids, amino acids and their derivatives, alkaloids, flavonoids, lipids, nucleotides and their derivatives, organic acids, lignans and coumarins, tannins, etc., which revealed different degrees of expression in the two rice cultivars at $30 \mathrm{DAF}$ compared to 0 DAF (Fig. 3a). Among the content of these metabolites, the content of organic acids and amino acids decreased at the later stage of ageing, and flavonoids, alkaloids and lipids accumulated more in YN at 30 DAF than in YB at 30 DAF. We then classified the different KEGG pathways (Supplemental Table S6). We analysed the top 20 metabolic pathways with the largest number of differentially accumulated metabolites (Fig. 3b, c) and found that, in the two rice cultivars, metabolites involved in the following accumulated more at 30 DAF than at 0 DAF: metabolic pathways; biosynthesis of secondary metabolites; ABC transporters; aminoacyl-tRNA biosynthesis; arginine and proline metabolism; 2-oxocarboxylic acid metabolism; alanine, aspartate and glutamate metabolism; flavonoid biosynthesis; etc. In recent years, we have noticed that secondary metabolism, such as phenylpropanoid metabolism and flavonoid metabolism, has been reported to be involved in reducing oxidative damage $e^{26}$. Our results are consistent with those in the literature, further suggesting that the substances involved in these metabolic processes may be involved in the regulation of plant senescence.

Integrated analysis reveals substantial changes in the flavonoid biosynthesis pathway during leaf senescence. To explore the differences in regulatory mechanisms underlying leaf senescence between the two rice cultivars, differentially accumulated metabolites and DEGs in KEGG pathways were selected for integrated analysis. We selected the metabolic pathways of differentially accumulated metabolites and their corresponding genes (Table 1). It is worth noting that the flavonoid synthesis pathway in both rice cultivars was significantly enriched in differentially accumulated metabolites and DEGs. We rearranged 10 major compounds of flavonoid biosynthesis and 5 DEGs to corresponding positions of the flavonoid biosynthesis pathway (Fig. 4a, b). In the flavonoid biosynthesis pathway, the abundance of 6 compounds increased at $30 \mathrm{DAF}$ compared with 0 DAF between the two cultivars (Fig. 4b, d). Our metabolic analysis revealed that flavonoids and flavonoid derivatives, including phloretin, luteolin and eriodictyol, showed lower abundances in YB than in YN at $30 \mathrm{DAF}$ (Fig. 4c, d). Secondary metabolites, including flavonoids, play an important role in maintaining redox homeostasis in cells by quenching reactive oxygen species ${ }^{27,28}$. The transcriptomic data showed that the relative expression of 5 DEGs (PAL, C4H, 4CL, CHS, CHI) in YN30/YN0 were generally higher than in YB30/YB0. To confirm this, we detected the expression of these five genes by qRT-PCR. The results were consistent with those obtained from RNA sequencing, the relative expression of $C 4 H, 4 C L$, CHS and $C H I$ were significantly increased, relative expression of $P A L$ increased slightly, but not significantly, indicating that the sequencing data sets are reliable. 


\begin{tabular}{|l|l|l|l|l|l|}
\hline \#Kegg_pathway & ko_id & Gene count & Meta count & Meta ID \\
\hline YN & ko01200 & 156 & 5 & C00979,C00025,C00049,C00234,C00122 \\
\hline Carbon metabolism & ko00480 & 72 & 1 & C00025 \\
\hline Glutathione metabolism & ko00941 & 64 & 4 & C01514,C00774,C05631,C09826 \\
\hline Flavonoid biosynthesis & ko00970 & 58 & 14 & $\begin{array}{l}\text { C00152,C00123,C00082,C00407,C00188,C00064,C00025,C00062,C00183,C00049,C00148,C00 } \\
\text { 135,C00073,C00234 }\end{array}$ \\
\hline Aminoacyl-tRNA biosynthesis & ko00051 & 39 & 1 & C00794 \\
\hline Fructose and mannose metabolism & ko00860 & 38 & 2 & C00188,C00025 \\
\hline Porphyrin and chlorophyll metabolism & ko00430 & 12 & 1 & C00025 \\
\hline Taurine and hypotaurine metabolism & & \multicolumn{1}{|l|}{} & \\
\hline YB & ko01200 & 171 & 4 & C00025,C00049,C00042,C00158 \\
\hline Carbon metabolism & ko00480 & 92 & 2 & C00025, C00315 \\
\hline Glutathione metabolism & ko00941 & 71 & 5 & C01514,C09826,C00774,C12136,C01477 \\
\hline Flavonoid biosynthesis & ko00051 & 51 & 1 & C00794 \\
\hline Fructose and mannose metabolism & ko00860 & 38 & 2 & C00188,C00025 \\
\hline Porphyrin and chlorophyll metabolism & & &
\end{tabular}

Table 1. Integrated analysis of differentially expressed genes and differentially accumulated metabolites associated with KEGG pathways.
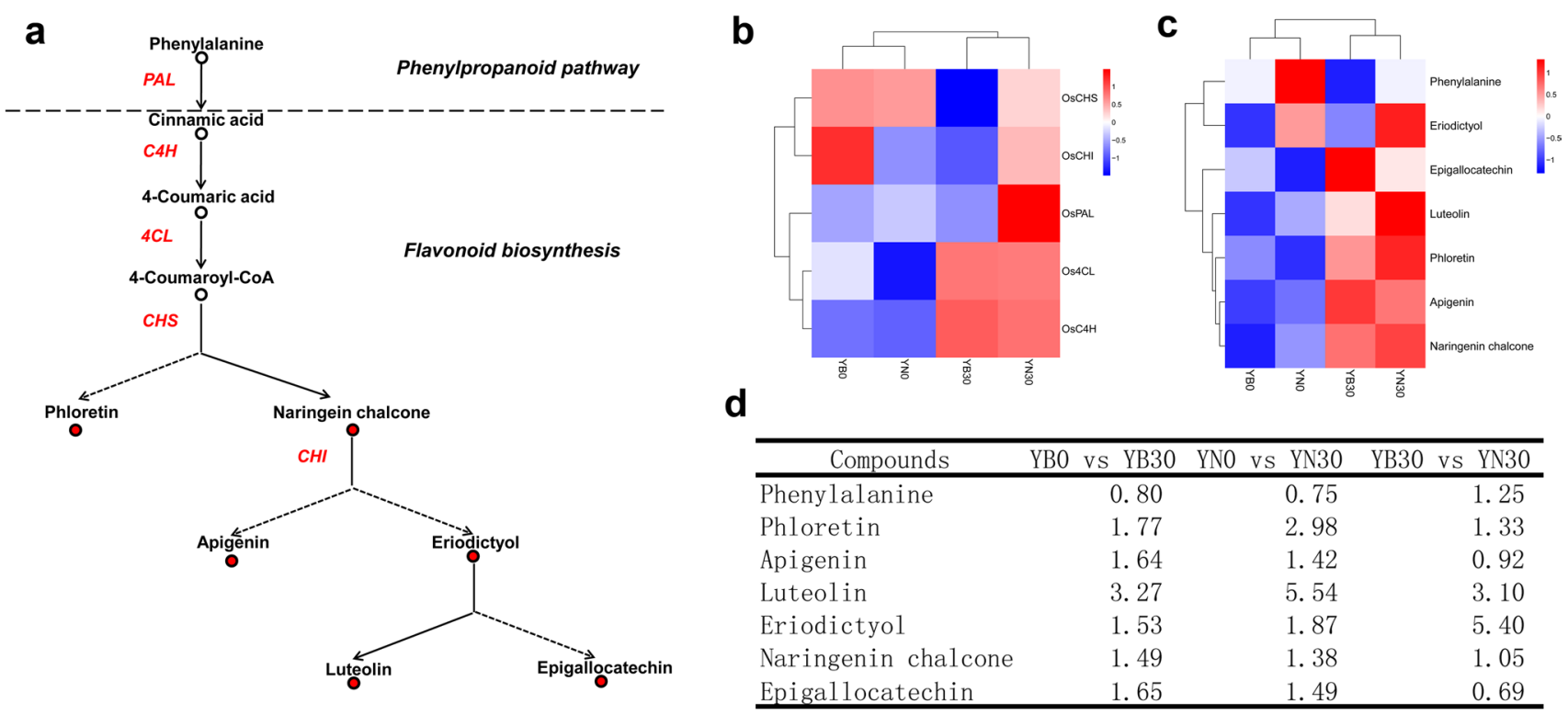

Figure 4. Differentially expressed genes and metabolites in the flavonoid biosynthesis pathway. (a) Representative genes and metabolites detected downstream of flavonoid biosynthesis. The red dots represent metabolites detected via metabolomics. $P A L$ phenylalanine ammonia-lyase, $C 4 H$ cinnamate 4 -hydroxylase, 4CL 4-coumaroyl-CoA:ligase, CHS chalcone synthase, CHI chalcone isomerase. (b) Heat map analysis of the expression of 5 genes in the flavonoid biosynthesis pathway. The data are homogenized by row. (c) Heat map analysis of 7 metabolites in the flavonoid biosynthesis pathway. The data are homogenized by row. (d) Metabolites detected in the flavonoid biosynthesis pathway.

(Supplemental Figure S4). Taken together, these results suggest that the increased accumulation of flavonoids may be due to alterations in the expression of these DEGs in YN.

A MYB transcription factor negatively regulates the expression of OsCHS and OsCHI. Transcription factors play important roles in plant growth, development, reproduction and senescence. As shown above, a large number of MYB transcription factors were differentially expressed at the later filling stage between the two rice cultivars and thus may be involved in important biological functions. Previous studies have reported that MYB TFs are involved in regulating the expression of genes involved in the flavonoid synthesis pathway in plants $^{29-31}$. According to our results, the expression of OsCHS and OsCHI, which encode chalcone synthase and chalcone isomerase, respectively, was significantly downregulated in YB at $30 \mathrm{DAF}$, which did not significantly differ from the results of YN (Supplemental Figure S4). We speculate that MYB TFs may be involved in the differential expression of $C H S$ and $C H I$ between the two rice varieties. To examine the relationship between MYBs 
a

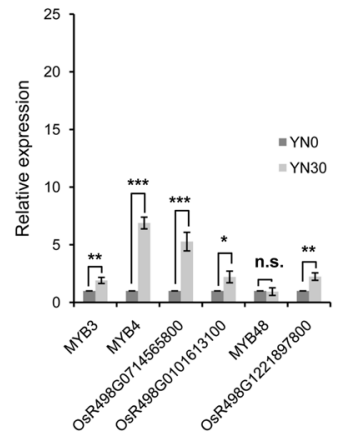

b

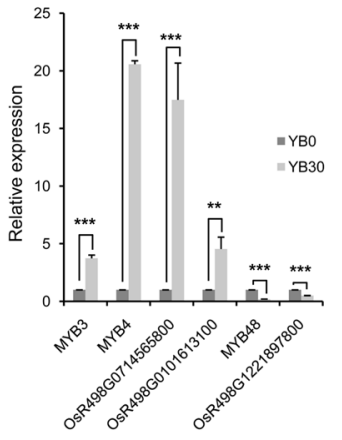

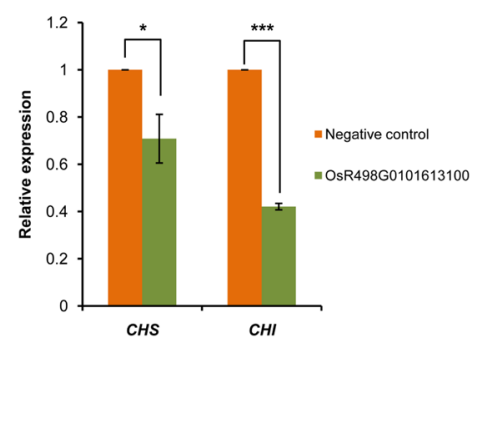

d

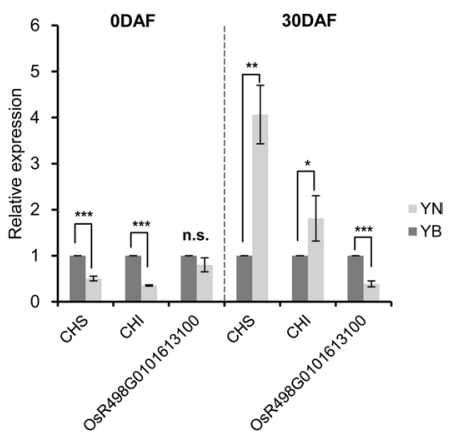

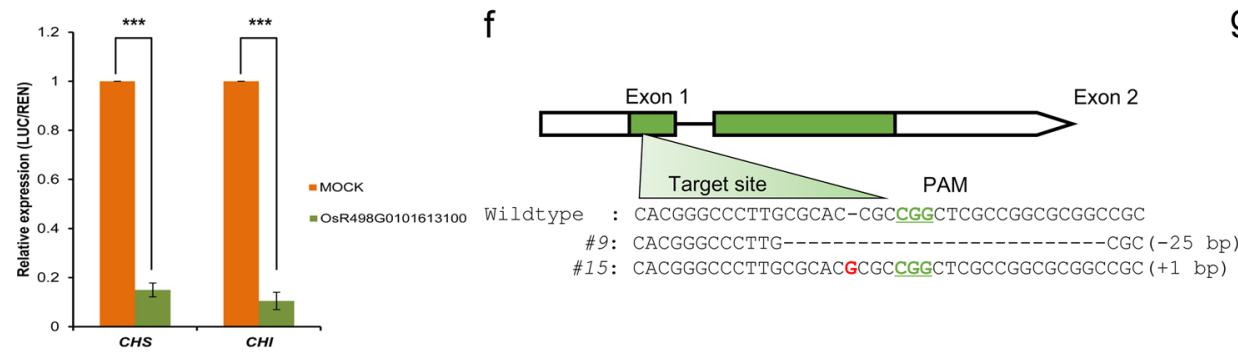

9

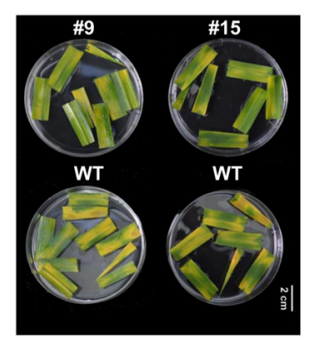

Figure 5. Expression regulation and anti-senescence analyses of MYB TFs. (a) qRT-PCR validation of MYB TFs in the YN0 versus YN30 comparison. (b) qRT-PCR validation of MYB TFs in the YB0 versus YB30 comparison. (c) qRT-PCR results of endogenous CHS and CHI genes in protoplasts transfected with OsR498G0101613100. Statistical analysis was performed using Student's t-test: ${ }^{\star}, P<0.05$; ${ }^{* *}, P<0.01$; ${ }^{* * *}$, $P<0.001$; n.s. not significant. (d) qRT-PCR validation of OsR498G0101613100, OsCHS and OsCHI between YN and YB. (e) Promoter activity analysis shows that OsR498G0101613100 significantly suppresses the transcriptional activity of CHS and CHI. ${ }^{* *}, P<0.001^{\star}$, $t$-test. (f) CRISPR-Cas9-mediated knockout of OsR498G0101613100. The green box represents the exon. Sequences of target in the CRISPR-Cas9 transgenic lines of rice variety Zhonghua11 (ZH11) were shown. Wild type refers to the sequence of ZH11. \#9, \#15 refer to the different types of knockout mutants. (g) Leaves phenotype after darkness treatment on the ko9 and ko15 mutants and the wild-type control ZH11. Photographs were taken 5 days after darkness treatment. Bar, $2 \mathrm{~cm}$.

and flavonoid synthesis, we first analysed and screened potential MYBs that positively or negatively regulated the expression of OsCHS and OsCHI. For example, the transcripts of OsR498G0102785500 (LOC_Os01g74410, MYB48) and OsR498G1221897800(LOC_Os12g37970), whose abundance decreased more significantly in YB at $30 \mathrm{DAF}$, may be involved in the positive regulation of flavonoid synthesis (Fig. 5a, b). By contrast, the transcripts of OsR498G0102429100 (LOC_Os01g65370, MYB3), OsR498G0408899800 (LOC_Os04g43680, MYB4), OsR498G0714565800(LOC_Os07g37210) and OsR498G0101613100(LOC_Os01g45090), whose abundance increased more significantly in $\mathrm{YB}$ at $30 \mathrm{DAF}$, may be involved in the negative regulation of flavonoid synthesis (Fig. 5a, b). We subsequently used protoplast transient assays to determine the activity of different MYB TFs in the activation of OsCHS and OsCHI transcription, with blank GFP vectors used as negative controls. The expression of endogenous OsCHS and $\mathrm{OsCHI}$ was significantly activated when upon transfection with the 35S:OsR498G0101613100 plasmid compared with other plasmids (Fig. 5c, Supplemental Figure S5). Comparison of OsR498G0101613100, OsCHS and OsCHI expression between YN and YB showed that expression of $\mathrm{OsCHS}$ and $\mathrm{OsCHI}$ were upregulated at $30 \mathrm{DAF}$ in the YB 30 versus YN 30 comparison, while expression of OsR498G0101613100 was downregulated at $30 \mathrm{DAF}$ in this comparison (Fig. 5d). In addition, the promoter activity analysis also showed that the presence of OsR498G0101613100 could significantly suppress the LUC/ REN ratio (Fig. 5e), suggesting that OsR498G0101613100 could essentially inhibit the transcriptional activity of OsCHS and OsCHI. In addition, we also generated knockout lines of OsR498G0101613100 in rice by using CRISPR-Cas9-mediated gene editing (Fig. 5f). Functional analysis of two homologous lines showed antisenescence phenotype after darkness treatment (Fig. 5g). These results indicated that OsR498G0101613100 plays a negative role in regulating the expression of $\mathrm{OsCHS}$ and $\mathrm{OsCHI}$, which may regulate the accumulation of metabolites in flavonoid biosynthesis pathway.

\section{Discussion}

High-quality rice with high yield, resistance, good quality and other good characteristics is the goal of rice breeding. Leaf colour transformation is one of important traits that been considered by breeders in the late stage of crop development, because it is actually a process of leaf senescence, in which the leaf colour changes from green to yellow, the photosynthetic capacity of leaves gradually decreases and the chlorophyll constantly degrades. It is estimated that the rice yield can be increased by $2 \%$ in theory if the life of functional leaves is prolonged one day at maturity stage ${ }^{32}$. Therefore, it is of great importance to cultivate anti-senescence rice varieties for stable and increased yield of rice. Yuenong Simiao is a conventional rice variety with high yield, disease resistance 
and senescence resistance, and therefore, is of practical significance for theoretical research and application of rice breeding to analyze its anti-senescence mechanism and explore the regulatory factors in the process of senescence.

Leaf senescence is the last stage of plant leaf development, but it is not the least important stage. During this period, a large number of functional genes and metabolites change rapidly, which makes the plant leaves turn from green to yellow, accompanied by the gradual transfer of nutrients to the seeds. Our study combined metabolomics with the transcriptomics to analyse metabolism-related genes and metabolites involved in the process of rice leaf senescence. We found that, at the late stage of ageing, the contents of organic acids and amino acids in the plants decreased, which was consistent with findings associated with plant ageing and development ${ }^{33}$. At the late stage of development, nitrogen is used for the sink/source transition, and the supply of nitrogen from leaves is weakened; the levels of the majority of amino acids and organic acids during this time have been studied ${ }^{16}$. On the other hand, at 30 DAF, the anti-senescence variety YN accumulated more flavonoids, alkaloids and lipids than did $\mathrm{YB}$, which may be due to the specificity of the YN variety. In the senescence process of Arabidopsis rosette leaves, contents decreased for chloroplast-localized lipid species; for example, the monogalactosyl diacylglycerols (MGDGs) and digalactosyl diacylglycerols (DGDGs) in the chloroplasts were degraded continuously during this process. Other lipid species, such as diacylglycerols, triacylglycerides (TAGs), ceramides, and glucosylceramides, and lysolipids such as lysophosphatidylethanolamines and lysophosphatidylcholines continuously increased during leaf senescence, which showed much higher accumulation in siliques than in senescent leaves ${ }^{34}$. In our study, the content of several free fatty acids increased at $30 \mathrm{DAF}$, including the following: lauric acid and 9,10-dihydroxy12-octadecenoic acid; some sphingolipids, such as D-erythro-dihydrosphingosine and 4-hydroxysphinganine; and lysophosphatidylcholines, such as LysoPC 17:0 and LysoPC 18:0. However, lysophosphatidylcholines such as LysoPC 16:0, LysoPC 18:4, and LysoPC 18:3 increased the most at 30 DAF in YN (Supplemental Table S5). These differences in lipid metabolite content patterns may be related to the difference in senescence patterns between monocotyledons and dicotyledons.

Through integrated analysis of transcriptional and metabolic data, we focused on changes in the flavonoid biosynthesis pathway. Flavonoids have been reported to be involved in reducing oxidative damage in plants ${ }^{35,36}$. The levels of flavonol glycosides and anthocyanins can be increased by overexpressing flavonoid structural genes such as CHS and DIHYDROFLAVONOL-4-REDUCTASE (DFR), which resulted in a reduction in ROS accumulation and enhanced tolerance to salt stress in rice ${ }^{37}$. In this pathway, phloretin, luteolin and eriodictyol, which were previously reported to be involved in attenuating oxidative stress, were detected to accumulate the most in YN 30 DAF (Fig. 4c, d) ${ }^{38-41}$. These compounds are antioxidant regulators in human and mouse cells, but their activity in plants has not been reported. Nevertheless, we speculate that these compounds may be associated with the anti-senescence phenotype of YN. However, apigenin, naringenin chalcone, and epigallocatechin also accumulated at 30 DAF. Due to their small differences between the two varieties, we did not focus on these compounds.

MYB TF family genes have been identified in different monocot and dicot species and have a wide range of important biological functions. MYB TFs are also involved in the regulation of plant flavonoid synthesis pathways in different species concerning the regulation of plant secondary metabolic responses. For example, AtMYB111, $A t M Y B 12$, and AtMYB11 are all independently capable of activating the expression of flavonol synthase (FLS), flavanone 3-hydroxylase $(F 3 H)$, chalcone isomerase $(C H I)$, and chalcone synthase $(C H S)^{42-44}$. GbMYBFL has been reported to enhance the accumulation of flavonoids ${ }^{45}$, DmMYB1 and CsMYB4a negatively regulate the synthesis of flavonoids ${ }^{46}$, GtMYBP3 and GtMYBP4 activate the expression of flavonol synthesis genes ${ }^{47}$, and so on. However, research on the relationship between MYBs and leaf senescence is limited. OsMYB102 inhibits ABA accumulation and downregulates ABA signalling responses to delay rice leaf senescence ${ }^{48}$. We found that the MYB TF OsR498G0101613100 may negatively regulate flavonoid biosynthesis to affect the antioxidant content. Furthermore, relationships between MYB TFs and the flavonoid pathway have been reported in citrus species. CsMYBF1 functions in controlling flavonol and hydroxycinnamic acid biosynthesis; interestingly, CsMYBF1 could activate the CHS gene promoter in Citrus but not in tomato ${ }^{49}$. We found that the MYB TF OsR498G0101613100 may inhibit the expression of $C H S$ and $C H I$, although whether this regulation occurs directly requires further experimental verification. $\mathrm{CHI}$ was previously reported to be involved in the regulation of hull and internode colour ${ }^{50}$. However, we detected the expression of $\mathrm{CHI}$ in flag leaves. Further functional experiments are needed to confirm whether OsR498G0101613100 regulates CHI directly during leaf senescence.

\section{Methods}

Plant materials and growth conditions. Leaf senescence assays were conducted under field conditions. For RNA-seq and gene expression analysis, various flag leaves were collected starting from the heading stage. To determine the precise expression patterns in $\mathrm{YN}$ and $\mathrm{YB}$, flag leaves were harvested every 10 days after flowering. To generate the knockout lines of OsR498G0101613100, the gRNA was designed to target the Exon 1 of this gene. The target sequence (+PAM) is 5'-CACGGGCCCTTGCGCACCGCCGG-3'. The constructs were then introduced into $\mathrm{pYLCRISPR/Cas9Pubi-H}$ vector and then transformed into the japonica rice Zhonghual1 cultivar by the Agrobacterium-mediated method ${ }^{51}$.

Chlorophyll and leaf SPAD measurements. Chlorophyll was extracted from $100 \mathrm{mg}$ of leaf tissue (fresh weight), after which its content was determined by measuring the absorbance at $470 \mathrm{~nm}, 645 \mathrm{~nm}$ and $663 \mathrm{~nm}$ using a Varioskan Flash spectrophotometer (Thermo Scientific) as described previously ${ }^{52}$. The SPAD values of the flag leaves were determined by a SPAD-502 $\mathrm{m}$. 
Darkness-induced leaf senescence. Fully expanded flag leaves were excised carefully. The detached leaves were cut into $3 \mathrm{~cm}$ pieces and floated on $25 \mathrm{ml}$ of water in Petri dishes, with the adaxial leaf side up. The samples were then incubated at $28^{\circ} \mathrm{C}$ in darkness for 5 days.

Expression analysis. Total RNA was extracted from leaf tissues or protoplasts using an RNAiso Plus Kit (Takara) according to the manufacturer's instructions. The RNA was reverse-transcribed using a Prime Script RT Reagent Kit together with gDNA Eraser (Takara). Quantitative real-time PCR was then performed using a TB Green Premix Ex Taq Kit (Takara) in conjunction with a CFX96 Real-Time PCR Detection System (BioRad) according to the manufacturer's instructions. Rice OsActin1 was used as an internal control. The primers used for quantitative RT-PCR (qRT-PCR) are listed in Supplemental Table S7. The values reported are the means \pm SDs of three biological repeats. Student's $t$-test was used for statistical analysis.

RNA-seq analysis. Total RNA was extracted from the flag leaves of rice plants at 0 DAF and 30 DAF. Three replicate RNA-seq libraries were prepared from YB0, YB30, YN0 and YN30. A total of 12 libraries were sequenced separately using a BGISEQ-500 sequencer (BGI-Shenzhen, China). Raw sequencing reads were cleaned by removing adaptor sequences, reads containing poly-N sequences, and low-quality reads. Approximately 40 M clean reads were mapped to the R498 reference genome (http://mbkbase.org/R498/) using HISAT2/ Bowtie 2 tools ${ }^{53,54}$. After the data were mapped, normalization was performed, and then FPKM (fragments per kilobase per million mapped reads) values were calculated using RESM software ${ }^{55}$. As previously described ${ }^{56}$, an adjusted $P$ value $\leq 0.001$ and a fold-change $\geq 2$ were used to identify differentially expressed genes (DEGs) in the YB0 versus YB30, YN0 versus YN30, YN0 versus YB0 and YN30 versus YB30 sample comparisons. GO (www. geneontology.org) and KEGG (www.kegg.jp) enrichment analyses of annotated differentially expressed genes were performed by phyper (https://en.wikipedia.org/wiki/Hypergeometric_distribution) based on the hypergeometric test. The significance levels of terms and pathways were corrected by $Q$ values with a rigorous threshold $(Q$ value $\leq 0.05)$ via Bonferroni corrections. TF annotation analysis was performed by getorf (http://emboss. sourceforge.net/apps/cvs/emboss/apps/getorf.html) and HMMsearch (http://hmmer.org).

Sample extraction and analysis of metabolites by ultra-performance liquid chromatography and tandem mass spectrometry. The flag leaves of rice plants at 0 DAF and 30 DAF were first freeze dried. Sample extract analysis, metabolite identification and quantification were performed at Wuhan MetWare Biotechnology Co., Ltd. (www.metware.cn), using a widely targeted metabolome method and in accordance with their standard procedures, as described in full previously ${ }^{57,58}$. Metabolite data analysis was conducted with Analyst 1.6.1 software (AB SCIEX, Ontario, Canada). The supervised multivariate method of partial least squares-discriminant analysis (PLS-DA) was used to maximize the metabolome differences between the pairs of samples. The relative importance of each metabolite to the PLS-DA model was checked using the variable importance in projection (VIP). Metabolites with a VIP $\geq 1$ and a fold-change $\geq 1.5$ or a fold-change $\leq 0.67$ were considered differentially accumulated metabolites for group discrimination in the YB0 versus YB30 and YN0 versus YN30 sample comparisons.

Integrated metabolome and transcriptome analysis. Differentially accumulated metabolites and DEGs in KEGG pathways were selected for integrated analysis. Differentially accumulated metabolites used for the integrated analysis were selected according to the information in Supplemental Table S6, and the DEGs used for the integrated analysis were selected from the top 15 metabolic pathways that were significantly enriched. We then analysed the overlapping metabolites and differentially expressed genes within these KEGG pathways.

Protoplast isolation and transfection. Ten-day-old rice plants were used for protoplast isolation according to a previously described procedure ${ }^{59,60}$. Briefly, the rice seedlings were cut into $0.5 \mathrm{~mm}$ strips and digested in an enzyme solution consisting of 1.5\% Cellulase R10, 0.4\% macerozyme R10, 0.4 M mannitol, 20 mM MES (pH 5.7), $20 \mathrm{mM} \mathrm{KCl}, 10 \mathrm{mM} \mathrm{CaCl}_{2}$ and $0.1 \%$ BSA for $3 \mathrm{~h}$. After mixing with $10 \mathrm{ml}$ of W5 solution consisting of $154 \mathrm{mM} \mathrm{NaCl}, 125 \mathrm{mM} \mathrm{CaCl}_{2}, 5 \mathrm{mM} \mathrm{KCl}$ and $2 \mathrm{mM}$ MES (pH 5.7), the digestion mixture was filtered through a Falcon cell strainer. Cells were collected by centrifugation for $2 \mathrm{~min}$ at $150 \times \mathrm{g}$, resuspended in $10 \mathrm{ml}$ of W5 solution and then incubated on ice for $30 \mathrm{~min}$. After centrifugation for $1 \mathrm{~min}$, the cell pellet was resuspended in $\mathrm{MMg}$ solution consisting of $0.4 \mathrm{M}$ mannitol, $15 \mathrm{mM} \mathrm{MgCl}_{2}$ and $4 \mathrm{mM} \mathrm{MES} \mathrm{(} \mathrm{pH}$ 5.7) to a concentration of $2 \times 10^{5}$ cells $/ \mathrm{ml}$. DNA transfection was carried out in a $2 \mathrm{ml}$ round-bottom microcentrifuge tube, where $200 \mu \mathrm{l}$ of protoplasts, $20 \mu \mathrm{l}$ of DNA $(2 \mu \mathrm{g} / \mu \mathrm{l})$, and $220 \mu \mathrm{l}$ of PEG solution (40\% PEG $4000(\mathrm{v} / \mathrm{v}), 0.2 \mathrm{M}$ mannitol and $0.1 \mathrm{M}$ $\mathrm{CaCl}_{2}$ ) were mixed together. The mixture was subsequently incubated at room temperature for 15 min before the transfection was quenched by adding $800 \mu \mathrm{l}$ of W5 solution. The transfected cells were harvested by centrifugation for $3 \mathrm{~min}$ at $150 \times \mathrm{g}$ and then incubated in a $1 \mathrm{ml} \mathrm{WI}$ solution consisting of $0.5 \mathrm{M}$ mannitol, $20 \mathrm{mM} \mathrm{KCl}$ and $4 \mathrm{mM}$ MES ( $\mathrm{pH}$ 5.7) in the dark for $12 \mathrm{~h}$ for RNA extraction.

Staining for hydrogen peroxide DAB-based analysis. Hydrogen peroxide was visually detected in leaves using DAB staining following the method described before ${ }^{61}$. The leaf samples were soaked in $1 \mathrm{mg} / \mathrm{ml}$ $3,3^{\prime}$-diaminobenzidine (DAB) for $8 \mathrm{~h}$ in the dark at $25^{\circ} \mathrm{C}$ before being heated in a boiling $95 \%$ ethanol bath for $10 \mathrm{~min}$ for destaining. Finally, the samples were equilibrated for $4 \mathrm{~h}$ in ethanol at $25^{\circ} \mathrm{C}$.

Promoter activity analysis. The $1384 \mathrm{bp}$ and $2000 \mathrm{bp}$ DNA fragments upstream of the starting codons of $\mathrm{CHS}$ and $\mathrm{CHI}$ genes, respectively, were amplified by polymerase chain reaction. The resulting promoter frag- 
ments of OsCHS and OsCHI were inserted into the pGreenII 0800-LUC vector to generate the reporter construct. The MYB transcriptional factor was fused with GFP in its C terminus, results in the effector construct of Ubi::OsR498G0101613100-GFP. The Ubi::GFP vector was used as the negative control. Different combinations of these above constructs were used for co-transfection in rice protoplasts. After co-transfection, the protoplasts were incubated at $28^{\circ} \mathrm{C}$ for $12 \mathrm{~h}$, and then total RNA were extracted. The reporter gene expression was calculated as relative ratio of $L U C$ to $R E N$ used qRT-PCR. Student's $t$-test was used for statistical analysis.

Statement on the use of plant. The authors declare that the use of plants parts in the present study complies with international, national and institutional guidelines.

Permissions statement. The authors declare that the collection of plant specimens comply with the IUCN Policy Statement on Research Involving Species at Risk of Extinction and the Convention on the Trade in Endangered Species of Wild Fauna and Flora.

Received: 19 February 2021; Accepted: 18 June 2021

Published online: 08 July 2021

\section{References}

1. Leng, Y., Ye, G. \& Zeng, D. Genetic dissection of leaf senescence in rice. Int. J. Mol. Sci. https://doi.org/10.3390/ijms18122686 (2017).

2. Hu, Y. et al. Jasmonate regulates leaf senescence and tolerance to cold stress: Crosstalk with other phytohormones. J. Exp. Bot. 68, 1361-1369. https://doi.org/10.1093/jxb/erx004 (2017).

3. Woo, H. R., Kim, H. J., Lim, P. O. \& Nam, H. G. Leaf senescence: Systems and dynamics aspects. Annu. Rev. Plant Biol. 70, $347-376$. https://doi.org/10.1146/annurev-arplant-050718-095859 (2019).

4. Pottier, M., Masclaux-Daubresse, C., Yoshimoto, K. \& Thomine, S. Autophagy as a possible mechanism for micronutrient remobilization from leaves to seeds. Front. Plant Sci. 5, 11. https://doi.org/10.3389/fpls.2014.00011 (2014).

5. Lim, P. O., Kim, H. J. \& Nam, H. G. Leaf senescence. Annu. Rev. Plant Biol. 58, 115-136. https://doi.org/10.1146/annurev.arplant. 57.032905.105316 (2007).

6. Nam, H. G. The molecular genetic analysis of leaf senescence. Curr. Opin. Biotechnol. 8, 200-207. https://doi.org/10.1016/s09581669(97)80103-6 (1997).

7. Li, Z., Peng, J., Wen, X. \& Guo, H. Gene network analysis and functional studies of senescence-associated genes reveal novel regulators of Arabidopsis leaf senescence. J. Integr. Plant Biol. 54, 526-539. https://doi.org/10.1111/j.1744-7909.2012.01136.x (2012).

8. Li, Z. et al. LSD 2.0: An update of the leaf senescence database. Nucl. Acids Res. 42, D1200-D1205. https://doi.org/10.1093/nar/ gkt1061 (2014).

9. Yu, Y. et al. Transcriptional landscape of pathogen-responsive lncRNAs in rice unveils the role of ALEX1 in jasmonate pathway and disease resistance. Plant Biotechnol. J. 18, 679-690. https://doi.org/10.1111/pbi.13234 (2020).

10. Breeze, E. et al. High-resolution temporal profiling of transcripts during Arabidopsis leaf senescence reveals a distinct chronology of processes and regulation. Plant Cell 23, 873-894. https://doi.org/10.1105/tpc.111.083345 (2011).

11. Woo, H. R. et al. Programming of plant leaf senescence with temporal and inter-organellar coordination of transcriptome in Arabidopsis. Plant Physiol. 171, 452-467. https://doi.org/10.1104/pp.15.01929 (2016).

12. Wang, W. et al. The involvement of cytokinin and nitrogen metabolism in delayed flag leaf senescence in a wheat stay-green mutant, tasg1. Plant Sci. 278, 70-79. https://doi.org/10.1016/j.plantsci.2018.10.024 (2019).

13. Li, W. et al. NAC family transcription factors in tobacco and their potential role in regulating leaf senescence. Front. Plant Sci. 9 , 1900. https://doi.org/10.3389/fpls.2018.01900 (2018).

14. Brown, A. V. \& Hudson, K. A. Developmental profiling of gene expression in soybean trifoliate leaves and cotyledons. BMC Plant Biol. 15, 169. https://doi.org/10.1186/s12870-015-0553-y (2015).

15. Akhter, D. et al. Transcriptional profile corroborates that BML mutant plays likely role in premature leaf senescence of rice (Oryza sativa L.). Int. J. Mol. Sci. https://doi.org/10.3390/ijms20071708 (2019).

16. Masclaux, C., Valadier, M. H., Brugiere, N., Morot-Gaudry, J. F. \& Hirel, B. Characterization of the sink/source transition in tobacco (Nicotiana tabacum L.) shoots in relation to nitrogen management and leaf senescence. Planta 211, 510-518. https://doi.org/10. 1007/s004250000310 (2000).

17. Himelblau, E. \& Amasino, R. M. Nutrients mobilized from leaves of Arabidopsis thaliana during leaf senescence. J. Plant Physiol. 158, 1317-1323. https://doi.org/10.1078/0176-1617-00608 (2001).

18. Nett, R. S., Lau, W. \& Sattely, E. S. Discovery and engineering of colchicine alkaloid biosynthesis. Nature 584, 148-153. https:// doi.org/10.1038/s41586-020-2546-8 (2020).

19. Lau, W. \& Sattely, E. S. Six enzymes from mayapple that complete the biosynthetic pathway to the etoposide aglycone. Science 349, 1224-1228. https://doi.org/10.1126/science.aac7202 (2015).

20. Szymanski, J. et al. Analysis of wild tomato introgression lines elucidates the genetic basis of transcriptome and metabolome variation underlying fruit traits and pathogen response. Nat. Genet. 52, 1111-1121. https://doi.org/10.1038/s41588-020-0690-6 (2020).

21. Tohge, T. From fruit omics to fruiting omics: Systematic studies of tomato fruiting by metabolic networks. Mol. Plant 13, 1114-1116. https://doi.org/10.1016/j.molp.2020.07.012 (2020).

22. Ma, X. et al. Transcriptomic and metabolomic studies disclose key metabolism pathways contributing to well-maintained photosynthesis under the drought and the consequent drought-tolerance in rice. Front. Plant Sci. 7, 1886. https://doi.org/10.3389/fpls. 2016.01886 (2016).

23. Zeng, D. et al. Rational design of high-yield and superior-quality rice. Nat. Plants 3, 17031. https://doi.org/10.1038/nplants.2017. 31 (2017).

24. Kong, Z., Li, M., Yang, W., Xu, W. \& Xue, Y. A novel nuclear-localized CCCH-type zinc finger protein, OsDOS, is involved in delaying leaf senescence in rice. Plant Physiol. 141, 1376-1388. https://doi.org/10.1104/pp.106.082941 (2006).

25. Guo, P. et al. A tripartite amplification loop involving the transcription factor WRKY75, salicylic acid, and reactive oxygen species accelerates leaf senescence. Plant Cell 29, 2854-2870. https://doi.org/10.1105/tpc.17.00438 (2017).

26. Dong, N. Q. \& Lin, H. X. Contribution of phenylpropanoid metabolism to plant development and plant-environment interactions. J. Integr. Plant Biol. https://doi.org/10.1111/jipb.13054 (2020). 
27. Dietz, K. J. Redox control, redox signaling, and redox homeostasis in plant cells. Int. Rev. Cytol. 228, 141-193. https://doi.org/10. 1016/s0074-7696(03)28004-9 (2003).

28. Heber, U., Lange, O. L. \& Shuvalov, V. A. Conservation and dissipation of light energy as complementary processes: Homoiohydric and poikilohydric autotrophs. J. Exp. Bot. 57, 1211-1223. https://doi.org/10.1093/jxb/erj104 (2006).

29. Ma, D., Reichelt, M., Yoshida, K., Gershenzon, J. \& Constabel, C. P. Two R2R3-MYB proteins are broad repressors of flavonoid and phenylpropanoid metabolism in poplar. Plant J. 96, 949-965. https://doi.org/10.1111/tpj.14081 (2018).

30. Wang, X. C. et al. Arabidopsis MYB4 plays dual roles in flavonoid biosynthesis. Plant J. 101, 637-652. https://doi.org/10.1111/tpj. $14570(2020)$.

31. Xu, W., Dubos, C. \& Lepiniec, L. Transcriptional control of flavonoid biosynthesis by MYB-bHLH-WDR complexes. Trends Plant Sci. 20, 176-185. https://doi.org/10.1016/j.tplants.2014.12.001 (2015).

32. Liu, D. H. The senescence of plant leaf. Plant Physiol. Commun. 2, 14-19 (1983) ((in Chinese)).

33. Balazadeh, S. et al. Reversal of senescence by N resupply to N-starved Arabidopsis thaliana: Transcriptomic and metabolomic consequences. J. Exp. Bot. 65, 3975-3992. https://doi.org/10.1093/jxb/eru119 (2014).

34. Watanabe, M. et al. Comprehensive dissection of spatiotemporal metabolic shifts in primary, secondary, and lipid metabolism during developmental senescence in Arabidopsis. Plant Physiol. 162, 1290-1310. https://doi.org/10.1104/pp.113.217380 (2013).

35. Jiang, N., Doseff, A. I. \& Grotewold, E. Flavones: From biosynthesis to health benefits. Plants (Basel) https://doi.org/10.3390/plant s5020027 (2016).

36. Zhan, X. et al. Rice sulfoquinovosyltransferase SQD2.1 mediates flavonoid glycosylation and enhances tolerance to osmotic stress. Plant Cell Environ. 42, 2215-2230. https://doi.org/10.1111/pce.13554 (2019).

37. Cui, L. G., Shan, J. X., Shi, M., Gao, J. P. \& Lin, H. X. The miR156-SPL9-DFR pathway coordinates the relationship between development and abiotic stress tolerance in plants. Plant J. 80, 1108-1117. https://doi.org/10.1111/tpj.12712 (2014).

38. Bai, J., Wang, Y., Zhu, X. \& Shi, J. Eriodictyol inhibits high glucose-induced extracellular matrix accumulation, oxidative stress, and inflammation in human glomerular mesangial cells. Phytother. Res. 33, 2775-2782. https://doi.org/10.1002/ptr.6463 (2019).

39. He, P. et al. Eriodictyol alleviates lipopolysaccharide-triggered oxidative stress and synaptic dysfunctions in BV-2 microglial cells and mouse brain. J. Cell. Biochem. 120, 14756-14770. https://doi.org/10.1002/jcb.28736 (2019).

40. Yang, S. C. et al. Luteolin attenuates neutrophilic oxidative stress and inflammatory arthritis by inhibiting Raf1 activity. Biochem. Pharmacol. 154, 384-396. https://doi.org/10.1016/j.bcp.2018.06.003 (2018).

41. Yang, Q. et al. Activation of Nrf2 by phloretin attenuates palmitic acid-induced endothelial cell oxidative stress via AMPKdependent signaling. J. Agric. Food Chem. 67, 120-131. https://doi.org/10.1021/acs.jafc.8b05025 (2019).

42. Pandey, A., Misra, P., Chandrashekar, K. \& Trivedi, P. K. Development of AtMYB12-expressing transgenic tobacco callus culture for production of rutin with biopesticidal potential. Plant Cell Rep. 31, 1867-1876. https://doi.org/10.1007/s00299-012-1300-6 (2012).

43. Pandey, A. et al. Co-expression of Arabidopsis transcription factor, AtMYB12, and soybean isoflavone synthase, GmIFS1, genes in tobacco leads to enhanced biosynthesis of isoflavones and flavonols resulting in osteoprotective activity. Plant Biotechnol. J. 12, 69-80. https://doi.org/10.1111/pbi.12118 (2014).

44. Misra, P. et al. Modulation of transcriptome and metabolome of tobacco by Arabidopsis transcription factor, AtMYB12, leads to insect resistance. Plant Physiol. 152, 2258-2268. https://doi.org/10.1104/pp.109.150979 (2010).

45. Zhang, W., Xu, F., Cheng, S. \& Liao, Y. Characterization and functional analysis of a MYB gene (GbMYBFL) related to flavonoid accumulation in Ginkgo biloba. Genes Genom. 40, 49-61. https://doi.org/10.1007/s13258-017-0609-5 (2018).

46. Zhu, L. et al. The heterologous expression of the chrysanthemum R2R3-MYB transcription factor CmMYB1 alters lignin composition and represses flavonoid synthesis in Arabidopsis thaliana. PLoS ONE 8, e65680. https://doi.org/10.1371/journal.pone.00656 80 (2013).

47. Nakatsuka, T. et al. Isolation and characterization of GtMYBP3 and GtMYBP4, orthologues of R2R3-MYB transcription factors that regulate early flavonoid biosynthesis, in gentian flowers. J. Exp. Bot. 63, 6505-6517. https://doi.org/10.1093/jxb/ers306 (2012).

48. Piao, W. et al. Rice transcription factor OsMYB102 delays leaf senescence by down-regulating abscisic acid accumulation and signaling. J. Exp. Bot. 70, 2699-2715. https://doi.org/10.1093/jxb/erz095 (2019).

49. Liu, C. et al. Characterization of a citrus R2R3-MYB transcription factor that regulates the flavonol and hydroxycinnamic acid biosynthesis. Sci. Rep. 6, 25352. https://doi.org/10.1038/srep25352 (2016).

50. Hong, L. et al. A mutation in the rice chalcone isomerase gene causes the golden hull and internode 1 phenotype. Planta 236, 141-151. https://doi.org/10.1007/s00425-012-1598-x (2012).

51. Nishimura, A., Aichi, I. \& Matsuoka, M. A protocol for Agrobacterium-mediated transformation in rice. Nat. Protoc. 1, 2796-2802. https://doi.org/10.1038/nprot.2006.469 (2006).

52. Porra, R. J., Schafer, W., Cmiel, E., Katheder, I. \& Scheer, H. The derivation of the formyl-group oxygen of chlorophyll b in higher plants from molecular oxygen. Achievement of high enrichment of the 7-formyl-group oxygen from ${ }^{18} \mathrm{O}_{2}$ in greening maize leaves. Eur. J. Biochem. 219, 671-679. https://doi.org/10.1111/j.1432-1033.1994.tb19983.x (1994).

53. Kim, D., Langmead, B. \& Salzberg, S. L. HISAT: A fast spliced aligner with low memory requirements. Nat. Methods 12, 357-360. https://doi.org/10.1038/nmeth.3317 (2015).

54. Langmead, B. \& Salzberg, S. L. Fast gapped-read alignment with Bowtie 2. Nat. Methods 9, 357-359. https://doi.org/10.1038/ nmeth.1923 (2012).

55. Li, B. \& Dewey, C. N. RSEM: Accurate transcript quantification from RNA-Seq data with or without a reference genome. $B M C$ Bioinform. 12, 323. https://doi.org/10.1186/1471-2105-12-323 (2011).

56. Wang, L., Feng, Z., Wang, X., Wang, X. \& Zhang, X. DEGseq: An R package for identifying differentially expressed genes from RNA-seq data. Bioinformatics 26, 136-138. https://doi.org/10.1093/bioinformatics/btp612 (2010).

57. Yuan, H. et al. Time-course comparative metabolite profiling under osmotic stress in tolerant and sensitive Tibetan hulless barley. Biomed. Res. Int. 2018, 9415409. https://doi.org/10.1155/2018/9415409 (2018).

58. Zhang, S. et al. Identification of potential metabolites mediating bird's selective feeding on Prunus mira flowers. Biomed. Res. Int. 2019, 1395480. https://doi.org/10.1155/2019/1395480 (2019).

59. Li, J. F., Zhang, D. \& Sheen, J. Epitope-tagged protein-based artificial miRNA screens for optimized gene silencing in plants. Nat. Protoc. 9, 939-949. https://doi.org/10.1038/nprot.2014.061 (2014).

60. Zhang, Y. et al. A highly efficient rice green tissue protoplast system for transient gene expression and studying light/chloroplastrelated processes. Plant Methods 7, 30. https://doi.org/10.1186/1746-4811-7-30 (2011).

61. Thordal-Christensen, H., Zhang, Z., Wei, Y. \& Collinge, D. Subcellular localization of $\mathrm{H}_{2} \mathrm{O}_{2}$ in plants. $\mathrm{H}_{2} \mathrm{O}_{2}$ accumulation in papillae and hypersensitive response during the barley-Powdery mildew interaction. Plant J. 11, 1187-1194. https://doi.org/10.1046/j. 1365-313X.1997.11061187.x (1997).

\section{Acknowledgements}

This research was supported by the grants from China Postdoctoral Science Foundation (2020M682639). Special Fund for Science and Technology Innovation Strategy (Construction of High-level Academy of Agricultural Sciences) (Foundation of President of Guangdong Academy of Agricultural Sciences in China, BZ201909). The national natural science foundation of China (31471175). Modern Agricultural Industry Technology System 
of Guangdong Province, China (2020KJ105). Guangdong Academy of Agricultural Sciences the 13th FiveYear Plan Subject team (201635TD). Research and development plan of key fields in Guangdong Province (2018B020202004-11). Provincial Rural Revitalization Strategy Project in 2019 (Yuecainong [2019]73). Research and development projects in key areas of Guangdong Province (2020B0202090003). Guangdong Provincial Key Laboratory (2020B1212060047).

\section{Author contributions}

X.H. conceived and supervised the study. J.X. and D.L. performed related experiments. The manuscript was written by J.X. under the supervision of X.H. W.L. and S.W. helped to analyzed the RNA-seq data. Z.L., X.W. and Z.F. helped to analyzed the metabolic data. All authors read and approved the final manuscript.

\section{Competing interests}

The authors declare no competing interests.

\section{Additional information}

Supplementary Information The online version contains supplementary material available at https:/doi.org/ 10.1038/s41598-021-93532-x.

Correspondence and requests for materials should be addressed to X.H.

Reprints and permissions information is available at www.nature.com/reprints.

Publisher's note Springer Nature remains neutral with regard to jurisdictional claims in published maps and institutional affiliations.

(1) Open Access This article is licensed under a Creative Commons Attribution 4.0 International License, which permits use, sharing, adaptation, distribution and reproduction in any medium or format, as long as you give appropriate credit to the original author(s) and the source, provide a link to the Creative Commons licence, and indicate if changes were made. The images or other third party material in this article are included in the article's Creative Commons licence, unless indicated otherwise in a credit line to the material. If material is not included in the article's Creative Commons licence and your intended use is not permitted by statutory regulation or exceeds the permitted use, you will need to obtain permission directly from the copyright holder. To view a copy of this licence, visit http://creativecommons.org/licenses/by/4.0/.

(C) The Author(s) 2021 